\title{
rworldmap: A New R package for Mapping Global Data
}

\author{
by Andy South
}

\begin{abstract}
rworldmap is a relatively new package available on CRAN for the mapping and visualisation of global data. The vision is to make the display of global data easier, to facilitate understanding and communication. The initial focus is on data referenced by country or grid due to the frequency of use of such data in global assessments. Tools to link data referenced by country (either name or code) to a map, and then to display the map are provided as are functions to map global gridded data. Country and gridded functions accept the same arguments to specify the nature of categories and colour and how legends are formatted. This package builds on the functionality of existing packages, particularly sp, maptools and fields. Example code is provided to produce maps, to link with the packages classInt, RColorBrewer and ncdf, and to plot examples of publicly available country and gridded data.
\end{abstract}

\section{Introduction}

Global datasets are becoming increasingly common and are frequently seen on the web, in journal papers and in our newspapers (for example a 'carbon atlas' of global emissions available at http: // image. guardian.co.uk/sys-files/Guardian/documents/ $2007 / 12 / 17 /$ CARBON_ATLAS.pdf). At the same time there is a greater interest in global issues such as climate change, the global economy, and poverty (as for example outlined in the Millenium Development Goals, http://www.un.org/millenniumgoals/bkgd. shtml). Thirdly, there is an increasing availability of software tools for visualising data in new and interesting ways. Gapminder (http://www.gapminder. org) has pioneered making UN statistics more available and intelligible using innovative visualisation tools and Many Eyes (http://www-958.ibm.com/) provides a very impressive interface for sharing data and creating visualisations.

World maps have become so common that they have even attracted satire. The Onion's Atlas of the Planet Earth (The Onion, 2007), contains a 'Bono Awareness' world map representing 'the intensity with which artist Bono is aware of the plight and daily struggles of region', with a categorisation ranging from 'has heard of nation once' through 'moved enough by nations crisis to momentarily remove sunglasses' to 'cares about welfare of nation nearly as much as his own'.
There appears to be a gap in the market for free software tools that can be used across disciplinary boundaries to produce innovative, publication quality global visualisations. Within $\mathrm{R}$ there are great building blocks (particularly sp, maptools and fields) for spatial data but users previously had to go through a number of steps if they wanted to produce world maps of their own data. Experience has shown that difficulties with linking data and creating classifications, colour schemes and legends, currently constrains researchers' ability to view and display global data. We aim to reduce that constraint to allow researchers to spend more time on the more important issue of what they want to display. The vision for rworldmap is to produce a package to facilitate the visualisation and mapping of global data. Because the focus is on global data, the package can be more specialised than existing packages, making world mapping easier, partly because it doesn't have to deal with detailed local maps. Through rworldmap we aim to make it easy for $\mathrm{R}$ users to explore their global data and also to produce publication quality figures from their outputs.

rworldmap was partly inspired and largely funded by the UK Natural Environment Research Council (NERC) program Quantifying Uncertainty in Earth System Science (QUEST). This program brings together scientists from a wide range of disciplines including climate modellers, hydrologists and social scientists. It was apparent that while researchers have common tools for visualisation within disciplines, they tend to use different ones across disciplines and that this limits the sharing of data and methods necessary for truly interdisciplinary research. Within the project, climate and earth system modellers tended to use IDL, ecologists ArcGIS, hydrologists and social scientists Matlab and fisheries scientists $R$. With the exception of $R$, these software products cost thousands of pounds which acts as a considerable constraint on users being able to try out techniques used by collaborators. This high cost and learning curve of adopting new software tools hinders the sharing of data and methods between disciplines. To address this, part of the vision for rworldmap was to develop a tool that can be freely used and modified across a multi-disciplinary project, to facilitate the sharing of scripts, data and outputs. Such freely available software offers greater opportunity for collaboration with research institutes in developing countries that may not be able to afford expensive licenses. 


\section{rworldmap data inputs}

rworldmap consists of tools to visualise global data and focuses on two types of data. Firstly, data that are referenced by country codes or names and secondly, data that are referenced on a grid.

\section{Country data}

There is a wealth of global country level data available on the internet including UN population data, and many global indices for, among others: Environmental Performance, Global Hunger and Multidimensional Poverty.

Data are commonly referenced by country names as these are the most easily recognised by users, but country names have the problem that vocabularies are not well conserved and many countries have a number of subtly different alternate names (e.g. Ivory Coast and Cote d'Ivoire, Laos and People's Democratic Republic of Lao). To address this problem there are ISO standard country codes of either 2 letters, 3 letters or numeric, and also 2 letter FIPS country codes, so there is still not one universally adopted standard. rworldmap supports all of these country codes and offers tools to help when data are referenced by names or other identifiers.

\section{Gridded data}

Global datasets are frequently spatially referenced on a grid, because such gridded or raster data formats offer advantages in efficiency of data storage and processing. Remotely sensed data and other values calculated from it are most frequently available in gridded formats. These can include terrestrial or marine data or both.

There are many gridded data formats, here I will concentrate on two: ESRI GridAscii and netCDF.

ESRI GridAscii files are an efficient way of storing and transferring gridded data. They are straightforward text files so can be opened by any text editor. They have a short header defining the structure of the file (e.g. number, size and position of rows and columns), followed by a single row specifying the value at each grid cell. Thus they use much less space than if the coordinates for each cell had to be specified.

Example start of gridAscii file for a half degree global grid:

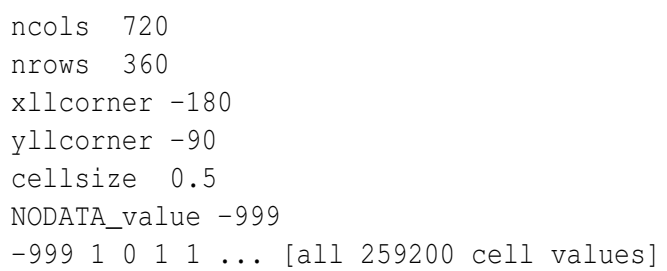

NetCDF is a data storage file format commonly used by climate scientists and oceanographers. NetCDF files can be multi-dimensional, e.g. holding $(x, y)$ data for multiple attributes over multiple months, years, days etc. The package ncdf is good for reading data from netCDF files.

\section{rworldmap functionality}

rworldmap has three core functions outlined below and others that are described later.

1. joinCountryData2Map() joins user country data referenced by country names or codes to a map to enable plotting

2. mapCountryData () plots a map of country data

3. mapGriddedData () plots a map of gridded data

\section{Joining country data to a map}

To join the data to a map use joinCountryData2Map. You will need to specify the name of column containing your country identifiers (nameJoinColumn) and the type of code used (joinCode) e.g. "ISO3" for ISO 3 letter codes or "UN" for numeric country codes.

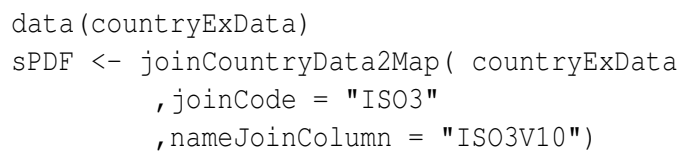

This code outputs, to the R console, a summary of how many countries are successfully joined. You can specify verbose $=$ TRUE to get a full list of countries. The object returned (named SPDF in this case) is of type "SpatialPolygonsDataFrame" from the package sp. This object is required for the next step, displaying the map.

If you only have country names rather than codes in your data, use joinCode="NAME"; you can expect more mismatches due to the greater variation within country names mentioned previously. To address this you can use the identifycountries () function described below, and change any country names in your data that do not exactly match those in the internal map.

\section{Mapping country data}

To plot anything other than the default map, mapcountryData requires an object of class "SpatialPolygonsDataFrame" and a specification of the name of the column containing the data to plot:

data (countryExData)

sPDF <- joinCountryData2Map ( countryExData , joinCode = "ISO3" , nameJoinColumn = "ISO3V10")

mapDevice() \#create world map shaped window mapCountryData (SPDF

, nameColumnToPlot= 'BIODIVERSITY' ) 


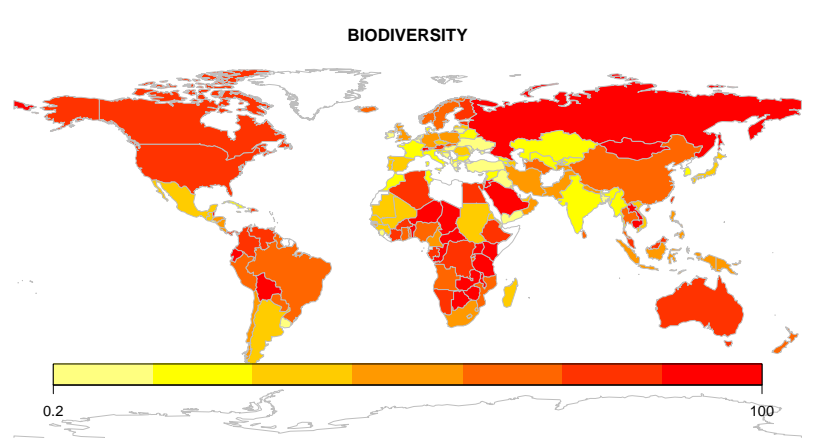

Figure 1: Output of mapCountryData()

\section{Mapping gridded data}

The mapGriddedData function can accept either

1. an object of type "SpatialGridDataFrame", as defined in the package sp

2. the name of an ESRI GridAscii file as a character string

3. a 2D R matrix or array (rows by columns)

rworldmap contains a "SpatialGridDataFrame" example that can be accessed and mapped as shown in the code and figure below.

data (gridExData)

mapDevice() \#create world map shaped window mapGriddedData (gridExData)

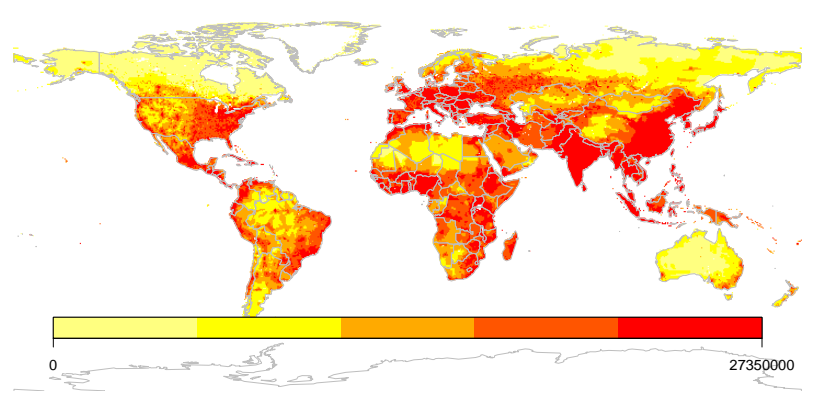

Figure 2: Output of mapGriddedData()

\section{Modifying map appearance}

rworldmap plotting functions are set up to work with few parameters specified (usually just those identifying the data) and in such cases, default values will be used. However, there is considerable flexibility to modify the appearance of plots by specifying values for arguments. Details of argument options are provided in the help files but here are a selection of the main arguments:

- catMethod determines how the data values are put into categories (then colourPalette determines the colours for those categories). Options for catMethod are: "pretty", "fixedWidth", "diverging", "logfixedWidth", "quantiles", "categorical", or a numeric vector defining the breaks between categories. Works with the next argument (numCats), although numCats is not used for "categorical", where the data are not modified, or if the user specifies a numeric vector, where the number of categories will be a result.

- numCats specifies the favoured number of categories for the data. The number of categories used may be different if the number requested is not possible for the chosen catMethod (e.g. for "quantiles" if there are only 2 values in the data it is not possible to have more than 2 categories).

- colourPalette specifies a colour palette to use from:

1. "palette" for the current palette

2. a vector of valid colours, e.g. c("red", "white", "blue") or output from RColorBrewer

3. a string defining one of the internal rworldmap palettes from: "heat", "diverging", "white2Black", "black2White", "topo", "rainbow", "terrain", "negpos8", "negpos9".

- addLegend set to TRUE for a default legend, if set to FALSE the function addMapLegend or addMapLegendBoxes can be used to create a more flexible legend.

- mapRegion a region to zoom in on, can be set to a country name from getMap () \$NAME or one of "eurasia", "africa", "latin america", "uk", "oceania", "asia".

\section{mapBubbles(), mapBars(), and mapPies()}

Another option for displaying data is to use the mapBubbles function which allows flexible creation of bubble plots on global maps. You can specify data columns that will determine the sizing and colouring of the bubbles (using namezSize and namezcolour). The function also accepts other spatialDataFrame objects or data frames containing columns specifying the $\mathrm{x}$ and $\mathrm{y}$ coordinates. If you wish to represent more attribute values per location there are also the newer mapBars () and mappies() functions to produce bar and pie charts respectively (noting that pie charts may not be the best way of presenting data when there are more than a few categories).

mapDevice() \#create world map shaped window mapBubbles $(\mathrm{dF}=$ getMap ()

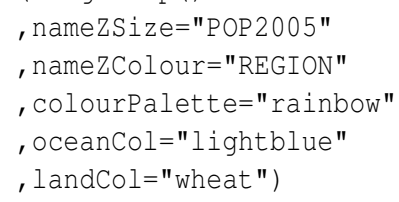




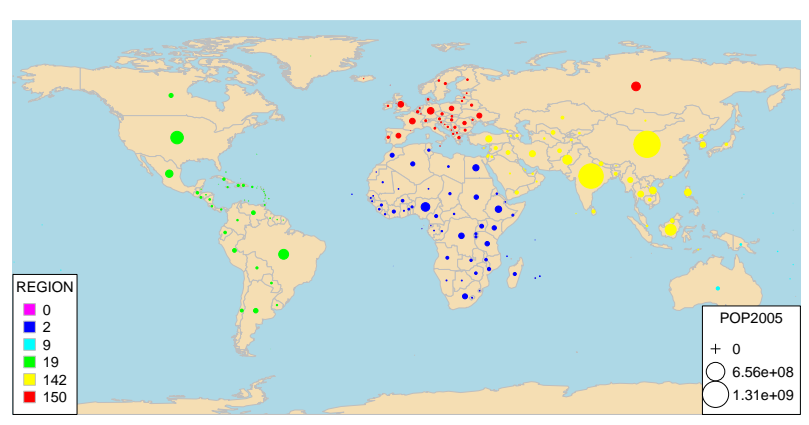

Figure 3: Output of mapBubbles()

\section{Identifying countries}

The interactive function identifyCountries() allows the user to click on the current map and, provided the cursor is sufficiently close to country centroid, will add the country name to the map. Optionally, the name of an attribute variable can also be passed to the function to cause the value of the attribute to be added to the label. e.g. 'Cape Verde 506807' will be printed on the map if the code below is entered and the mouse clicked over those islands (if you know where they are!).

identifyCountries (getMap () , nameColumnToPlot="POP2005" )

\section{Aggregating data to produce different out- puts}

rworldmap offers options for aggregating half degree gridded data to countries, and in turn for aggregating country level data to regions. In both of these options a range of aggregation options are available including mean, minimum, maximum and variance.

mapHalfDegreeGridToCountries() takes a gridded input file, aggregates to a country level and plots the map. It accepts most of the same arguments as mapCountryData ().

Country level data can be aggregated to global regions specified by regionType in country2Region() which outputs as text, and mapByRegion() which produces a map plot. The regional classifications available include SRES (The Special Report on Emissions Scenarios of the Intergovernmental Panel on Climate Change (IPCC) ), GEO3(Global Earth Observation), Stern and GBD (Global Burden of Disease).

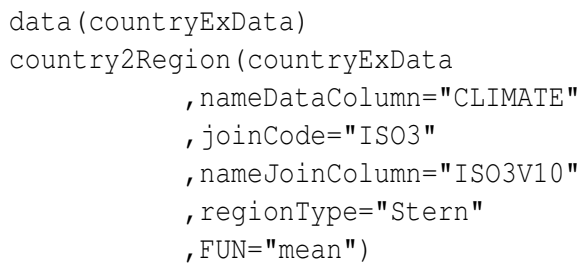

Outputs this text:

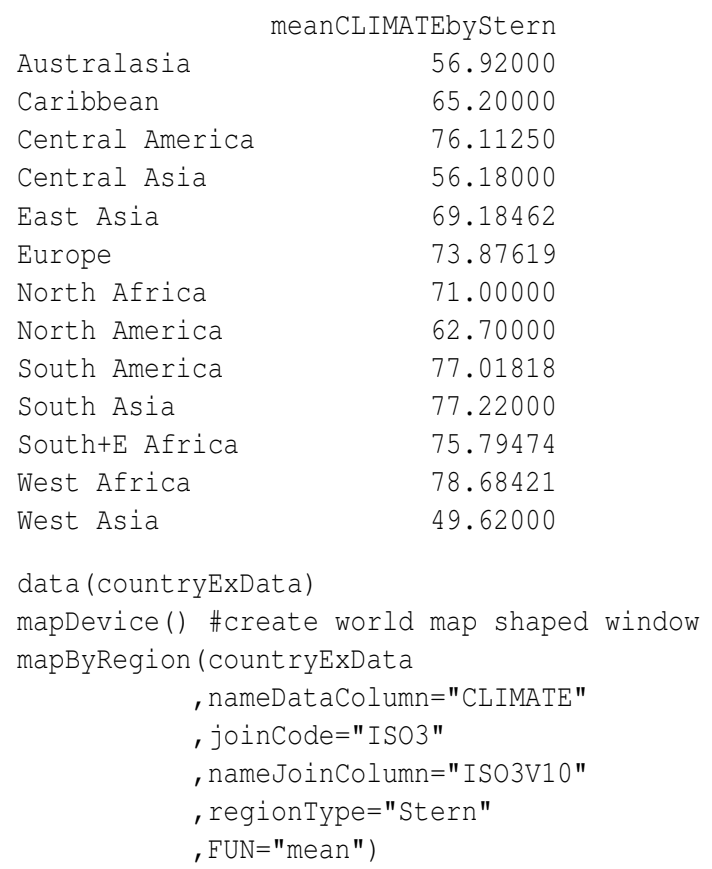

Produces this map: mean CLIMATE by Stern regions

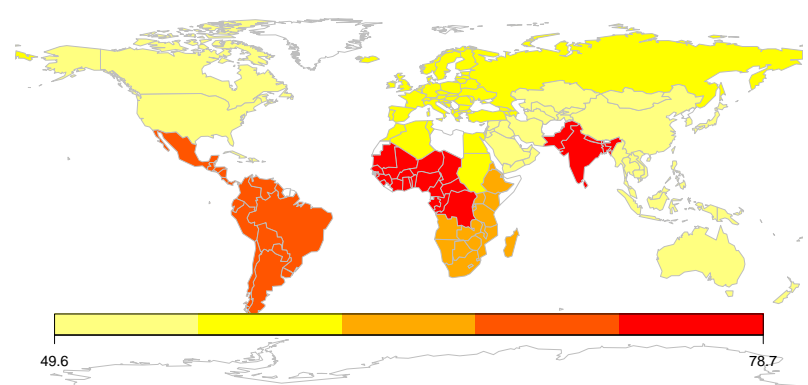

Figure 4: Output of mapByRegion()

The identity of which countries are in which regions are stored in the data frame countryRegions. This also identifies which countries are currently classed by the UN as Least Developed Countries (LDC), Small Island Developing states (SID) and Landlocked Developing Countries (LLDC). To map just the Least Developed Countries the code below could be used:

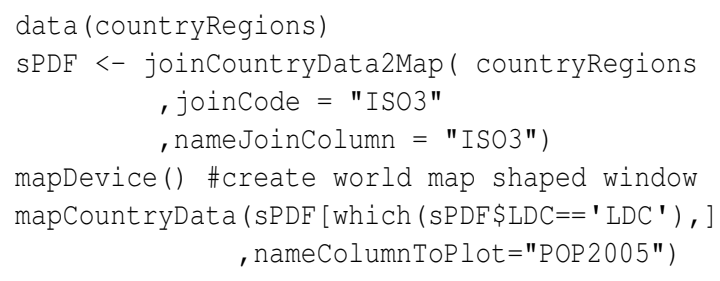

\section{Using rworldmap with other packages classInt and RColorBrewer}

While rworldmap sets many defaults internally there are also options to use other packages to have greater flexibility. In the example below classInt is used to create the classification and RColorBrewer to specify the colours. 

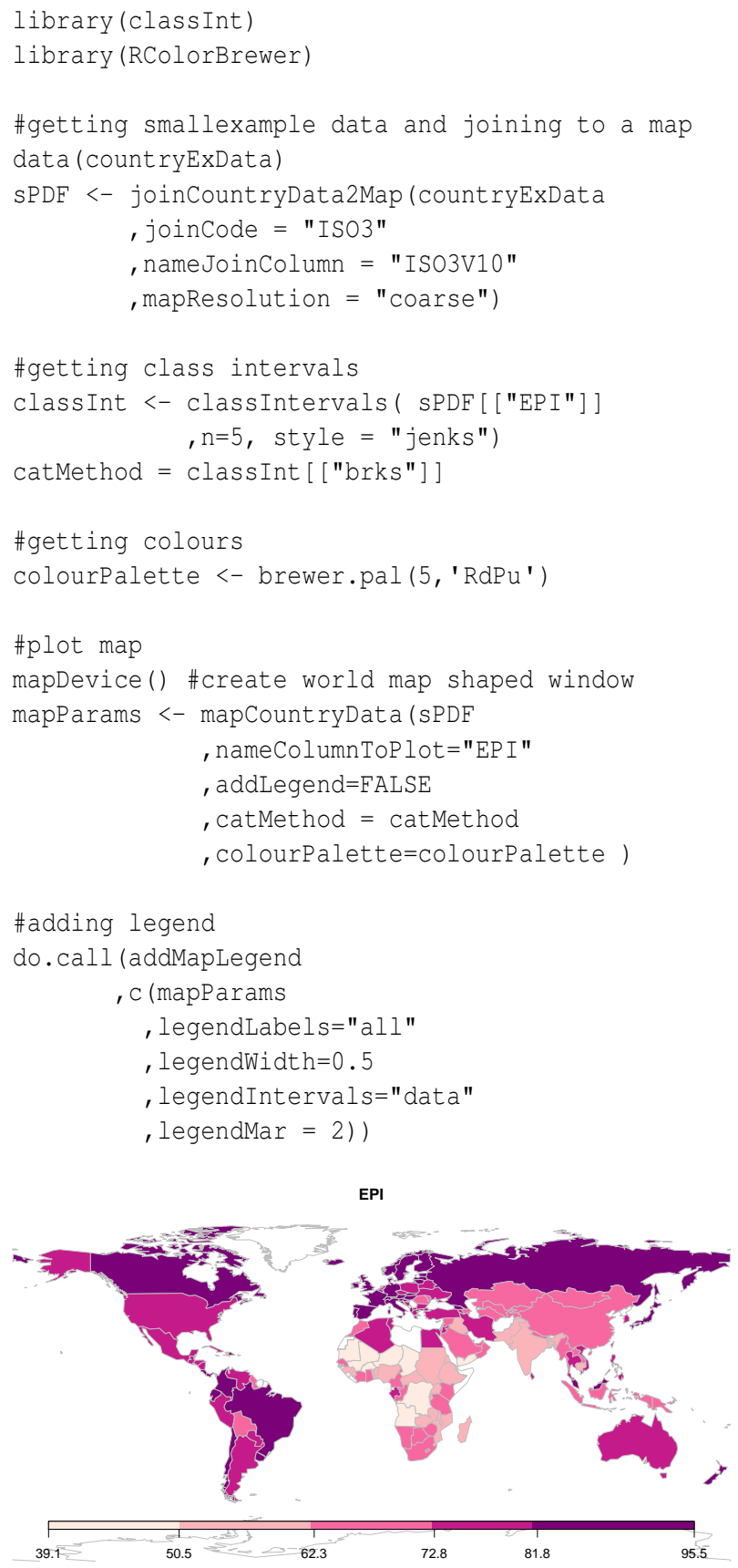

Figure 5: Output of mapcountryData () using inputs from classInt and RColorBrewer

\section{Examples using data freely avail- able on the web}

\section{Example country data from the web}

The Happy Planet Index or HPI (http://www. happyplanetindex.org) combines country by country estimates of life expectancy, life satisfaction and ecological footprint to create an index of sustainable well-being that makes much more sense than GDP for assessing how countries are doing (Marks, 2010).

An Excel file containing the data ('hpi-2-0results.xIs') can be downloaded from the HPI website at: http://www.happyplanetindex.org/ learn/download-report.html.

I copied the country data from the sheet 'All Data' and pasted into a '.csv' file so that the header row was at the top of the file and the summary statistics at the bottom were left off. I then edited some of the column names to make them appropriate $\mathrm{R}$ variable names (e.g. changing 'Life Sat (0-10)' to 'LifeSat').

This data can then be read in easily using read.csv() and joinCountryData2Map ().

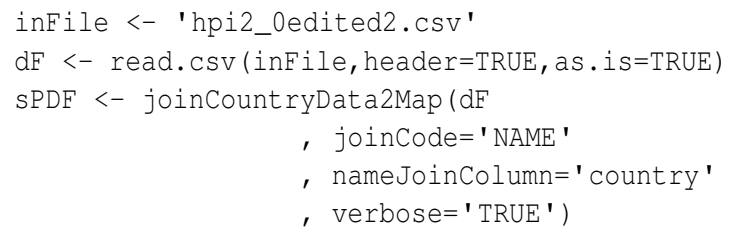

Unfortunately, but in common with many global country level datasets, the countries are specified by name alone rather than by ISO country codes. The verbose=TRUE option in joinCountryData2Map () can be used to show the names of the 10 countries that don't join due to their names being slightly different in the data than rworldmap. The names of the countries that failed to join can be edited in the csv to be the same as those in getMap () [ ['NAME'] ], and then they will join. A selection is shown below.

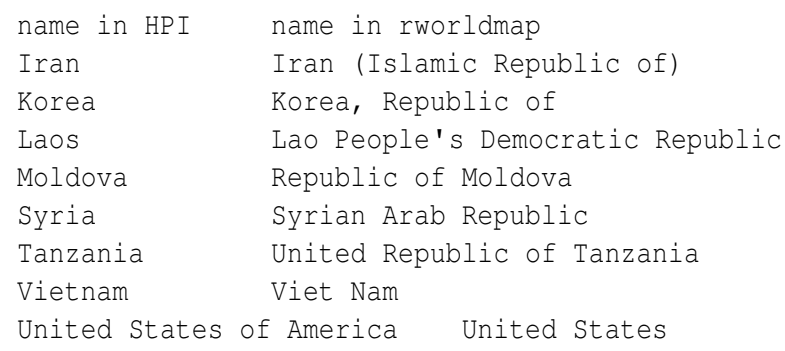

The map of the HPI on the website is interesting in that the colours applied to countries are not determined by the value of the HPI for that country, but instead by the values of the three component indices for Life Expectancy, Life Satisfaction and Ecological Footprint. Therefore I needed to add some extra R code to be able to recreate the HPI map.

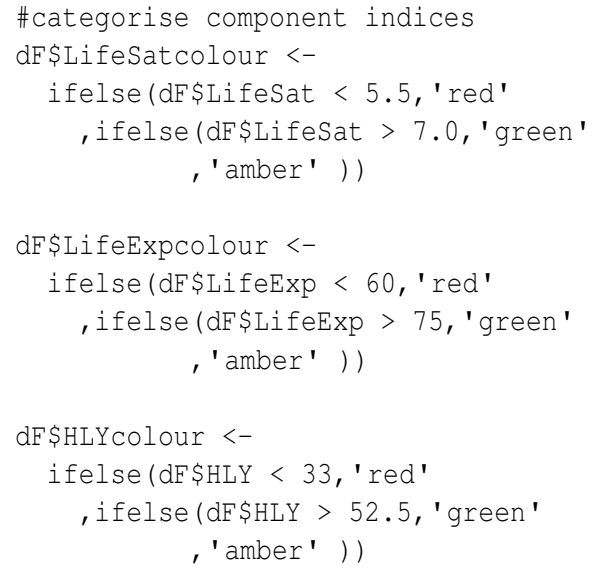




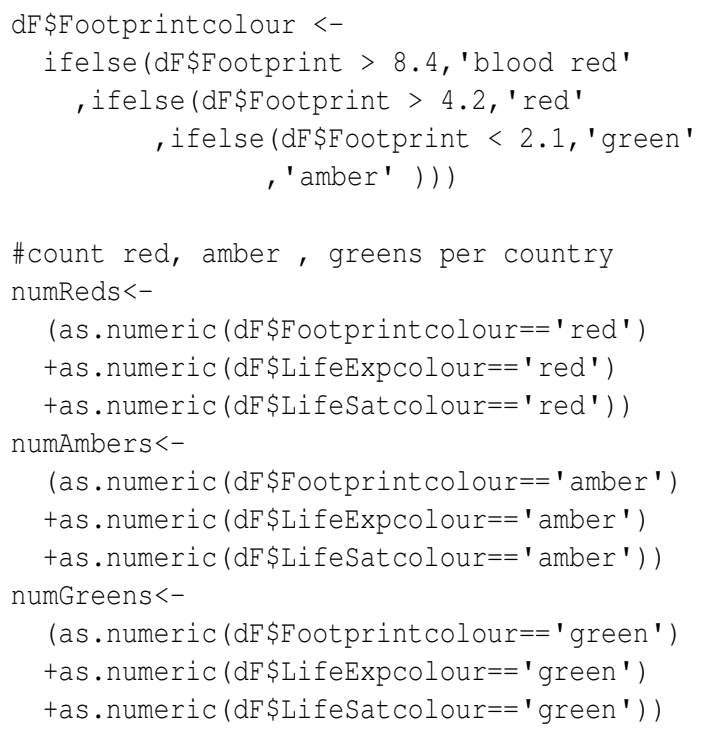

\#changing legendText

mapParams\$legendText <-

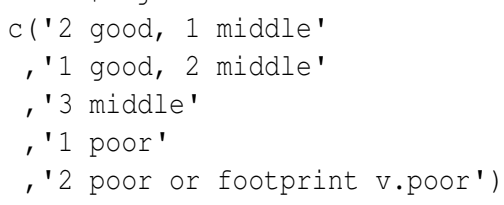

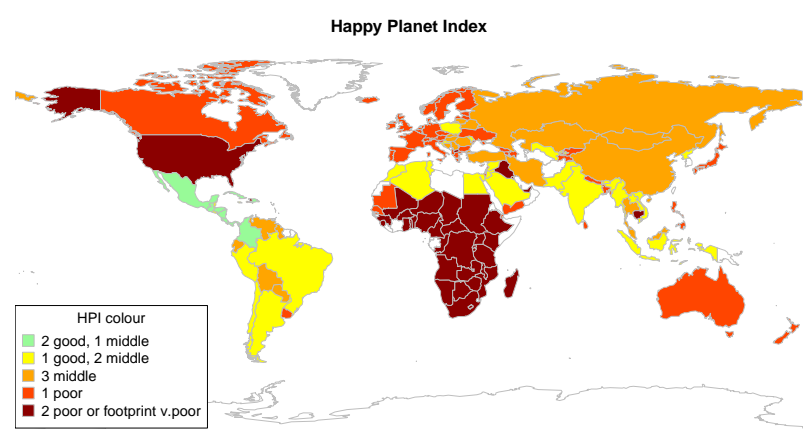

Figure 6: Happy Planet Index 2.0, using rworldmap to replicate map in happy planet report

\section{Example gridded data from the web}

'Koeppen Geiger' is a published classification dividing the world into 30 climatic zones. The GIS files for a Koeppen Geiger gridded climatic regions map are freely available from $h t t p: / /$ koeppen-geiger. vu-wien.ac.at/. The code below shows how to read in and plot an ascii file downloaded from that site.

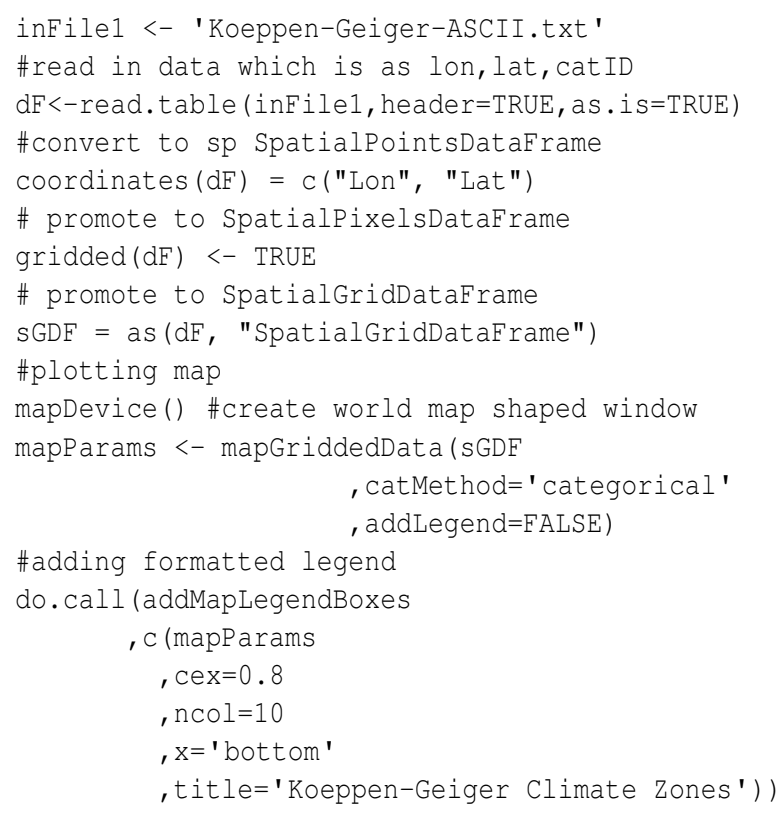

This produces a map that looks a lot different from the published map because it is using a different colour palette. The default "heat " colour palette is not the best for this categorical data and one of the palettes from RColorBrewer more suitable for categorical data could be used. However it would be good to retain the palette created by the authors of the data. The ascii file does not contain any colour information, however the authors also provide ESRI and Google Earth compatible files that do have colour information. It appeared to be impossible to extract the palette from the ESRI files, but by opening the '.kmz' file in Google Earth, saving to '. $\mathrm{kml}$ ' and some fiddly text editing in $\mathrm{R}$ the colours 
could be extracted as a single column then saved to a '.csv' file, the first few lines of which are: colour

\#960000

\#ff0000

\#ff9999

\#ffcccc

Plotting the map in the original colours can then be achieved relatively simply by:
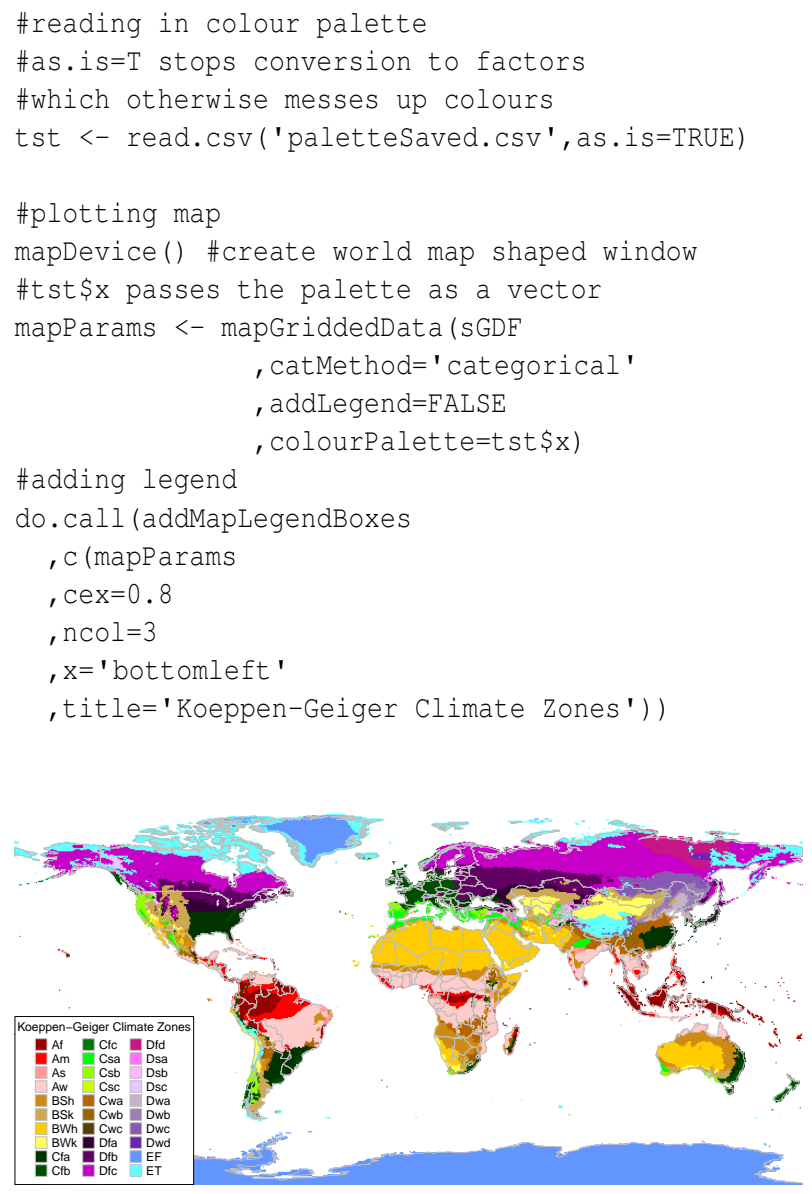

Figure 7: 'Koeppen Geiger' climatic zones, map produced using mapGriddedData () and freely available data

\section{Using rworldmap to map netCDF data in tandem with ncdf}

netCDF is a common file format used in meteorology and oceanography, it is both multi-dimensional and self documented. The package ncdf allows netCDF files to be opened, queried and data extracted directly to $R$. Data extracted from netCDF files in this way can then be converted to sp objects and plotted using rworldmap. In the example below the netCDF file $(\sim 333 \mathrm{~KB})$ is first downloaded from the IPCC data distribution centre at: http://www.ipcc-data. org/cgi-bin/downl/ar4_nc/tas/HADCM3_SRA1B_1_ tas-change_2046-2065.cyto180.nc.
This particular file was accessed from: http:// www.ipcc-data.org/ar4/info/UKMO-HADCM3_SRA1B_ tas.html

The ' lon180' option was chosen to get a file with longitude values from -180 to 180 as that requires less editing before plotting.

\section{library (ncdf)}

\#the downloaded file

infile <-

'HADCM3_SRA1B_1_tas-change_2046-2065.cyto180.nc' memory.limit(4000) \#set memory limit to max

$\mathrm{nc}=$ open.ncdf (inFile, write=FALSE)

print (nc) prints to console a description of the file contents which includes the information shown below (edited slightly for brevity).

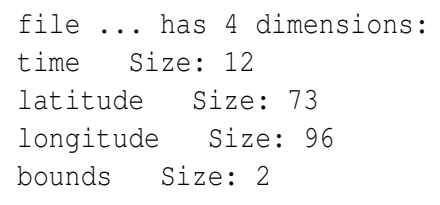

air_temperature_anomaly[longitude, latitude, time]

After the netCDF file has been opened in this way, selected variables can be read into $R$ using get.var.ncdf(), converted to a SpatialGridDataFrame and plotted using the rworldmap function mapGriddedData. The code below first creates a grid from the parameters in netCDF file, and is written to be generic so that it should work on other similar netCDF files. Then it creates a standard classification and colour scheme for all of the plots based on having looked at the values in each months data first. Finally it loops through all months reads in the data for that month, creates a spatialGridDataFrame for each, plots it and saves it as a '.png'.

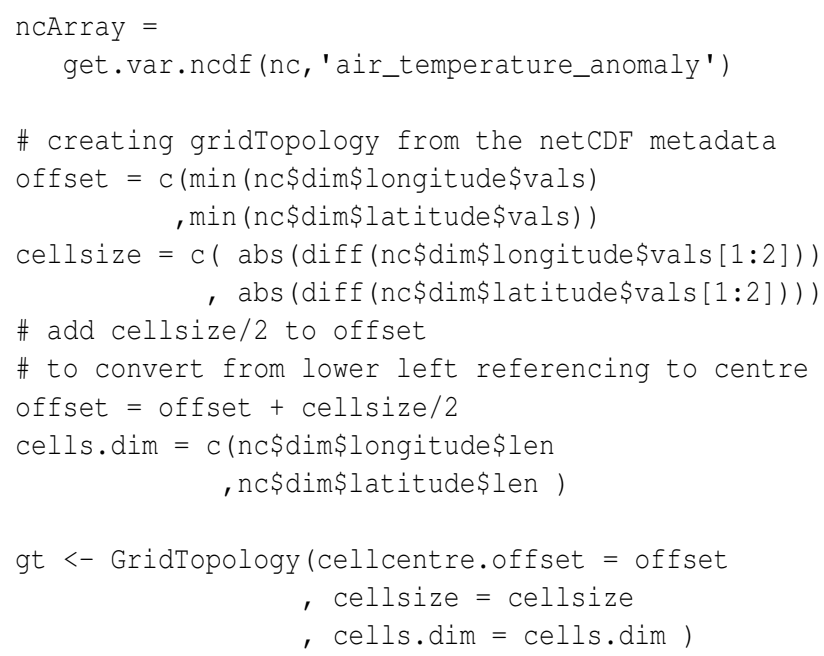



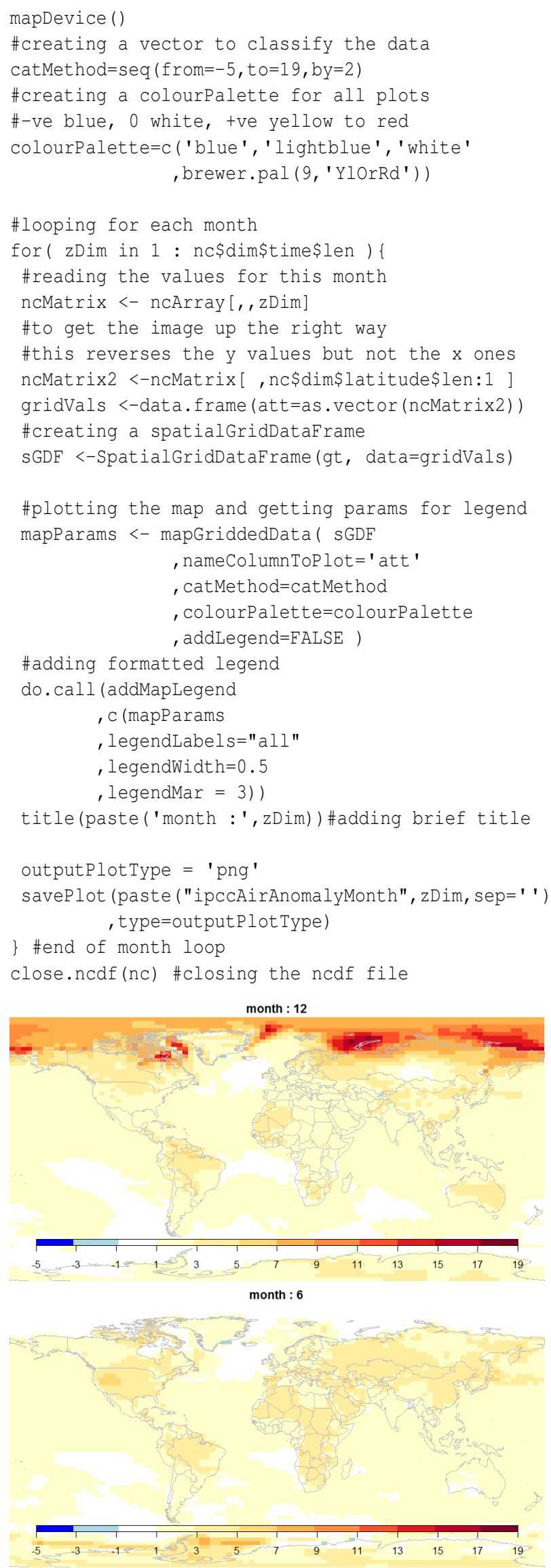

Figure 8: Two examples of IPCC temperature anomaly data (for December and June respectively) plotted using mapGriddedData ()

\section{Summary}

rworldmap is a new package to facilitate the display of global data, referenced by grid, country or region. It is available on CRAN at http://cran.r-project. org/web/packages/rworldmap. rworldmap aims to provide tools to improve the communication and understanding of world datasets. If you have any comments or suggestions or would like to contribute code please get in touch. The source code and development versions are available from http: / code. google.com/p/rworld/. I plan to extend this work by making datasets, including those used in this paper, more easily accessible, perhaps through a package called rworldmapData. We are also working on visualisations to communicate more information than by maps alone. For more help on rworldmap there is a vignette available at http: / / cran.r-project.org/ web/packages/rworldmap/vignettes/rworldmap. pdf and an FAQ at http://cran.r-project.org/ web/packages/rworldmapvignettes/rworldmapFAQ. pdf.

\section{Acknowledgements}

This work was funded by NERC through the QUEST Data Initiative (QESDI) and QUEST Global Systems Impacts (GSI) projects. Thanks to Pru Foster and Sarah Cornell at QUEST and Graham Pilling at Cefas without whose enthusiasm this would not have happened. Thanks to Martin Juckes for understanding project management, Barry Rowlingson and Roger Bivand for their contributions at a startup workshop and after, and to Nick Dulvy, Joe Scutt-Phillips and Elisabeth Simelton for inspiration on global vulnerability analyses and colour.

\section{Bibliography}

N. Marks GDP RIP (1933-2010). Significance, 7(1):27-30, 2010. Published Online: 23 Feb 2010 URL http://www3.interscience.wiley. com/cgi-bin/fulltext/123300797/PDFSTART.

The Onion Our Dumb World: The Onion's Atlas of the Planet Earth. Little, Brown, and Company, 2007. ISBN: 978075289120 0. URL http: //www. theonion. com/.

Andy South

Centre for Environment, Fisheries and Aquaculture Science (Cefas)

Pakefield Road, Lowestoft, NR33 OHT, UK

Current address: Graduate School of Education

University of Exeter

Exeter, EX1 2LU, UK

southandy@gmail.com 


\section{Appendix: Comparing using rworldmap to not using it}

Here I show a brief comparison between using rworldmap and not. It is not my intention to set up a straw-man that demonstrates how superior my package is to others. Other packages have different objectives, by focusing on world maps I can afford to ignore certain details. It is only by building on the great work in other packages that I have been able to get this far. Those caveats aside here is the comparison using some data on alcohol consumption per adult by country, downloaded as an Excel file from the gapminder website at http://www . gapminder.org/data/ and saved as a '.csv'. Reading in the data is common to both approaches:

infile <- 'indicatoralcoholconsumption20100830.csv' $d F<-$ read.csv(infile)

\section{Using rworldmap}

library (rworldmap)

sPDF <- joinCountryData2Map (dF,

, joinCode = "NAME"

, nameJoinColumn = "X"

, nameCountryColumn = "X"

, verbose $=$ TRUE)

mapCountryData (sPDF, nameColumnToPlot=' X2005' )

\section{Not using rworldmap}

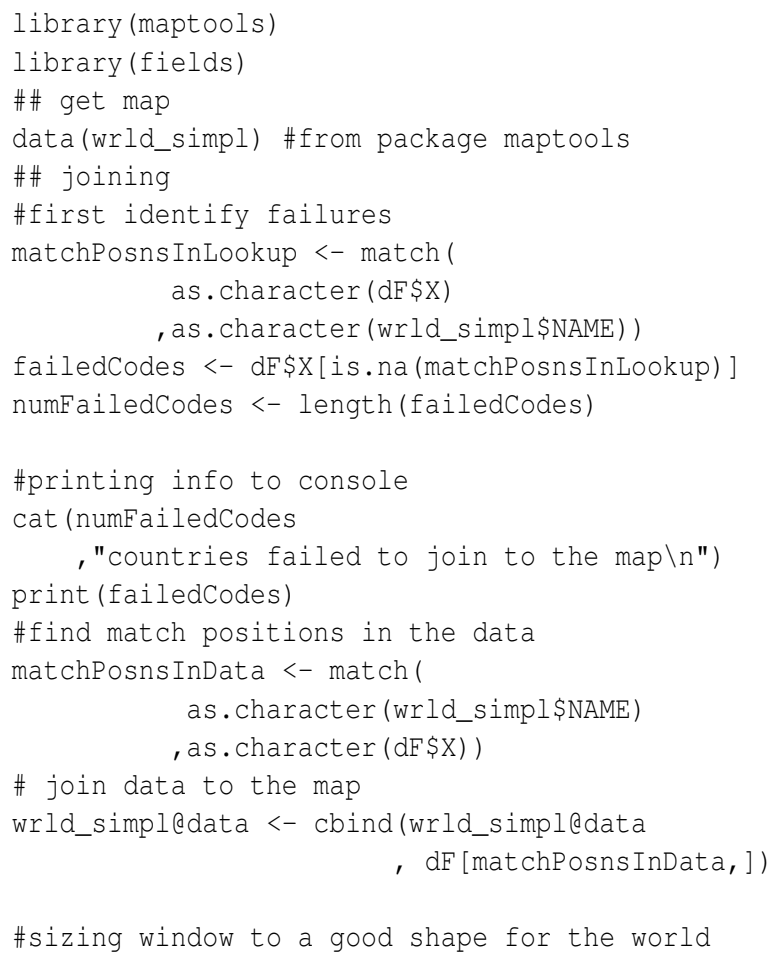

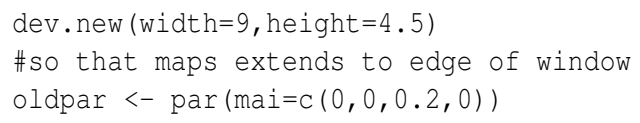

par(oldpar) \#reset graphics settings

Slight differences in country naming, and an absence of country codes, causes 9 countries not to to join in both approaches. joinCountryData2Map () outputs the identities of these countries. This means that in the maps it incorrectly looks like alcohol consumption is zero in both the UK and USA. To correct this the country names need to be renamed prior to joining, either in the '.csv' or in the dataframe. In the $R$ dataframe the countries could be renamed by:

n1<-'United Kingdom of Great Britain and Northern Ireland' n2<-'United Kingdom'

levels $(\mathrm{dF} \$ \mathrm{X})$ [which (levels $(\mathrm{dF} \$ \mathrm{X})==\mathrm{n} 1)$ ] $<-\mathrm{n} 2$

The objective for rworldmap is to make world mapping easier, based on difficulties experienced by the author and project collaborators. Of course, there is still a learning curve associated with being able to use rworldmap itself. All of the operations shown in this paper can be accomplished by accomplished $\mathrm{R}$ programmers without the need for rworldmap, however in an inter-disciplinary project rworldmap made it easier for scientists who are new to $R$ to start producing outputs, and encouraged them to extend their $\mathrm{R}$ learning further. There are repetitive data manipulation routines that are tricky to implement, rworldmap reduces the time spent on these so that more time can be spent on the important task of communicating what the data say. 